\title{
Tracking harmful chemicals and pathogens using the Human Health Observatory at ASU
}

\section{Rolf U. Halden, Elizabeth Terlinden, Simona Kraberger, Matthew Scotch, Joshua Steele, Arvind Varsani}

Biodesign Center for Environmental Health Engineering, Arizona State University, Tempe, Arizona, United States

\section{Objective}

To highlight the new science of population and urban metabolism metrology, for characterizing human exposures to biological agents, narcotics, antimicrobials and other contaminants of emerging concern using community wastewater as a diagnostic matrix.

\section{Introduction}

Sewerage systems of villages, townships, cities and megacities convey the urine, stool, blood, and sputum of community members, enabling through analysis of community wastewater, a near real-time assessment of population health status and of emerging public health threats. Signature compounds and biomarkers targeted analytically for surveillance may include chemical and biological threat agents, transformation products of the same, human metabolites, biomarkers of exposure and other markers of interest. Additional information can be gleaned by analyzing, in a similar fashion, municipal sewage sludge resulting from wastewater treatment, a material that during treatment becomes enriched in persistent, hydrophobic and potentially bioaccumulative pollutants, while more biodegradable compounds are converted to methane mostly. When taken together, these multi-dimensional data sources promise to yield critical information on the health status, sustainability and resilience of rural and urban human populations in a new scientific approach termed population metabolism metrology, or for city environments, urban metabolism metrology.

\section{Methods}

Starting in 2001, samples of wastewater and municipal sludge were collected from cities across the United States and from around the world. Obtained samples were logged, archived and split samples analyzed for chemical and biological markers of human health concern. Sampling and analyses are ongoing. The sample archive is a shared resource available to the international research community.

\section{Results}

Over the course of $15+$ years, wastewater and municipal sludges from over 300 cities around the world have been collected (see Figure 1 below). The resultant expansive specimen archive today is known as the Human Health Observatory (HHO) at Arizona State University. It constitutes the largest repository of wastewater process flow samples in the world. The municipal sludge samples contained in the Human Health Observatory constitute the U.S. National Sewage Sludge Repository (NSSR) and international Sewage Sludge Repository (iSSR). Archived samples have been analyzed for over 300 chemical and biological threat agents, resulting in U.S. national inventories of dozens of harmful chemicals, time- and space-resolved insights into human exposure to pesticides and substances of abuse, the identification of chemical threats fostering drug resistance and antibiotic crossresistance, and the discovery of hundreds of novel viruses of potential human health importance. Public health and policy events informed by the HHO and NSSR include the U.S. federal ban of antimicrobials in personal care products regulated by the U.S Food and Drug Administration, and the Florence Statement of Triclosan and Triclocarban, a global call for elimination of unnecessary and ineffective antimicrobials from consumer products.

\section{Conclusions}

The analysis of wastewater and municipal sewage sludges collected at centralized wastewater treatment facilities around the world represents an effective, inexpensive and rapid approach for public health assessment and threat detection. Among the notable success stories thus far are the 2017 US FDA ban of multiple antimicrobials whose overuse has been linked to the emergence of drug resistance and cross-resistance of human pathogens to antibiotics used in human medicine. 
ISDS 2019 Conference Abstracts

\section{Acknowledgement}

This project was supported in part by grant numbers 1R01ES015445, R01ES020889, their multiple supplements from the National Institute of Environmental Health Sciences (NIEHS) and by grant number LTR 05/01/12 from the Virginia G. Piper Charitable Trust.

\section{References}

1. Magee HY, Maurer MM, Cobos A, Pycke BFG, Venkatesan AK, et al. 2018. US nationwide reconnaissance of ten infrequentlymonitored antibiotics in municipal biosolids. Sci Total Environ. 643, 460-67. PubMed https://doi.org/10.1016/j.scitotenv.2018.06.206

2. Halden RU. Invited Plenary Talk: Urban metabolism metrology: a new discipline elucidating the human condition in cities around the world, $252^{\text {nd }}$ American Chemical Society's National Meeting, Philadelphia, PA, August 21-25, 2016.

3. Venkatesan AK, Done HY, Halden RU. 2015. United States National Sewage Sludge Repository at Arizona State University-a new resource and research tool for environmental scientists, engineers, and epidemiologists. Environ Sci Pollut Res Int. 22(3), 1577-86. PubMed https://doi.org/10.1007/s11356-014-2961-1

4. Venkatesan AK, Halden RU. Wastewater Treatment Plants as Chemical Observatories to Forecast Ecological and Human Health Risks of Manmade Chemicals. Sci Rep-Uk 2014, 4.

5. Muhire BM, Varsani A, Martin DP. 2014. SDT: A Virus Classification Tool Based on Pairwise Sequence Alignment and Identity Calculation. PLoS One. 9(9), e108277. PubMed https://doi.org/10.1371/journal.pone.0108277

6. Cheung KH, Yip KY, Townsend JP, Scotch M. 2008. HCLS 2.0/3.0: Health care and life sciences data mashup using Web 2.0/3.0. J Biomed Inform. 41(5), 694-705. PubMed https://doi.org/10.1016/j.jbi.2008.04.001 


\section{ISDS 2019 Conference Abstracts}
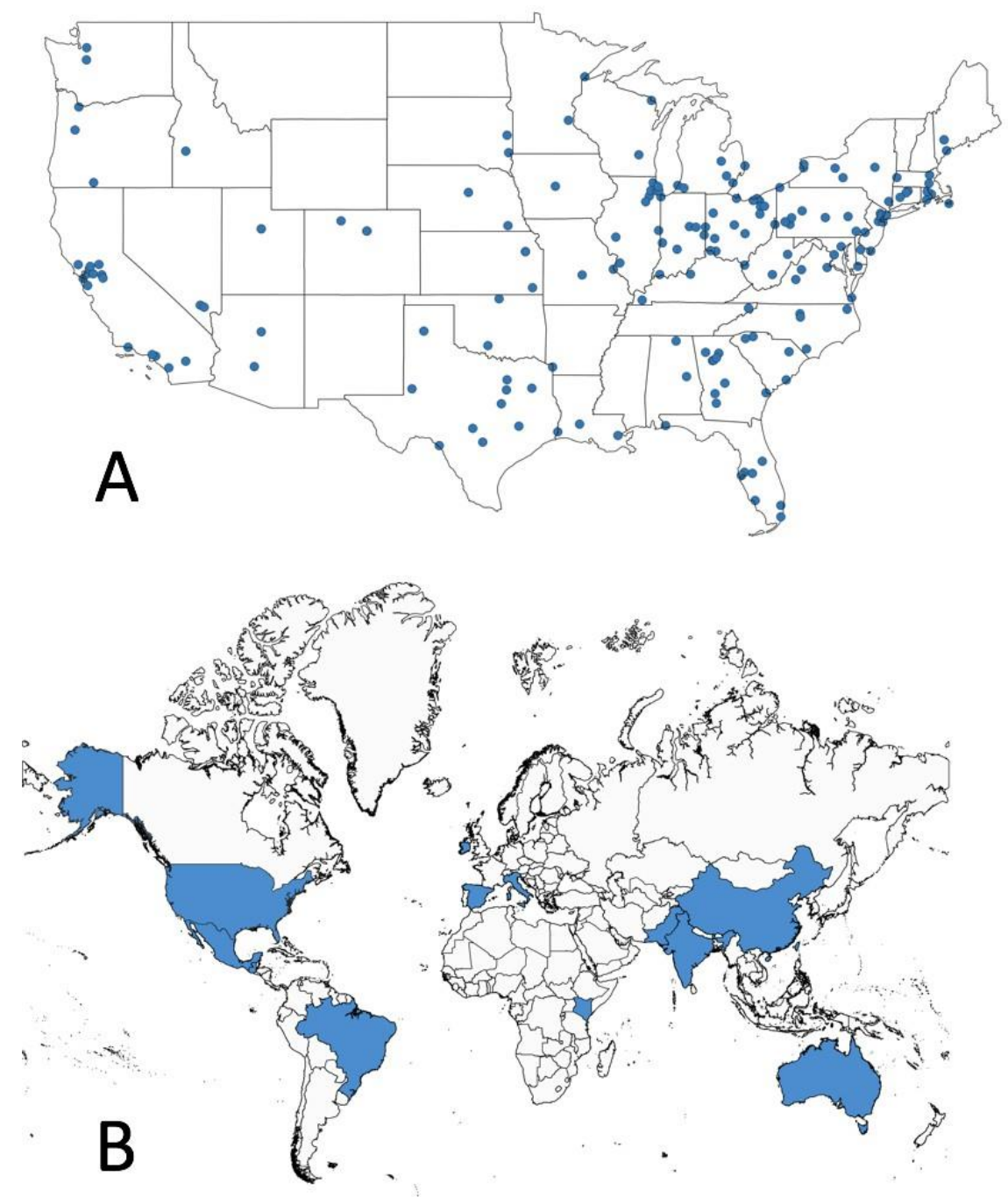

Figure 1. U.S. national (A) and international sampling locations (B) represented in the Human Health Observatory (HHO) at Arizona State University. 